\title{
BIRDS OF A CENTRAL SÃO PAULO WOODLOT: 1. CENSUSES 1982-2000
}

\author{
WILLIS, E. O. ${ }^{1,2}$ and ONIKI, Y. ${ }^{2}$ \\ ${ }^{1,2}$ Departamento de Zoologia, Unesp, Campus de Rio Claro, \\ C.P. 199, CEP 13506-900, Rio Claro, SP, Brazil \\ ${ }^{2}$ Instituto de Estudos da Natureza, Rua 2, no 2272, CEP 13500-153, Rio Claro, SP, Brazil \\ Correspondence to: Edwin O’Neill Willis, Instituto de Biologia, Departamento de Zoologia, Unesp, C.P. 199, \\ CEP 13506-900, Rio Claro, SP, Brasil, e-mail: ewillis@ rc.unesp.br \\ Received July 31, 2000 - Accepted February 5, 2001 - Distributed May 31, 2002
}

\begin{abstract}
Some 263 birds were recorded near and in a 230-ha patch of semideciduous forest in cane fields of central São Paulo, Brazil. Subtracting 67 open-area species, 22 of marshes or creeks, 10 vagrants and 12 recorded later, 152 forest and border species were recorded in 1982-86, much like what was observed in a similar woodlot near Campinas. Both woodlots lost species gradually over the years. Some birds avoided hard cane-field edges, preferring soft bushy edges. Of open-area species, 22 seemed to have disappeared by 1997 due to earlier high El Niño rains or rare permanently open habitats in the sugar cane; 17 new species were mostly nocturnal ones not noted earlier, or occasional visitors. Forest and borders lost 31 species, gaining five of dry regions and one winter visitor. Several migrants from the south appeared only in wet years before recent greenhouse effects, some resident birds were hunted, and canopy hummingbirds were perhaps still present. Dry-forest travel-prone or "metapopulational" species moved their centers of distribution, partly concealing loss of moist-forest diversity.
\end{abstract}

Key words: birds, conservation, greenhouse effect, metapopulations, semideciduous forest, sugar cane.

\section{RESUMO}

\section{Aves de uma mata na região central de São Paulo: 1. Censos 1982-2000}

Foram listadas 263 aves próximo e dentro de uma mata semidecídua de 230 ha em meio a plantações de cana-de-açúcar, na região central do Estado de São Paulo, Brasil. Subtraindo-se 67 espécies de áreas abertas, 22 de riachos, 10 vagantes e 12 observadas mais tarde, cerca de 152 espécies de mata e de borda foram observadas entre 1982-86, semelhantemente às observadas em uma mata próxima a Campinas. Ambas as matas perderam espécies gradualmente. Algumas aves evitaram as margens "duras" dos canaviais, preferindo as margens "macias" arbustivas. Das espécies de área aberta, parece que 22 desapareceram até 1997, em razão das abundantes chuvas anteriores causadas por El Niño, ou pela raridade de habitats permanentemente abertos nos canaviais; 17 espécies "novas" eram principalmente noturnas que não haviam sido checadas anteriormente, ou visitantes ocasionais. A mata e as bordas perderam 37 espécies, ganhando 5 de regiões secas e 1 visitante de inverno. Vários migrantes do sul apareceram somente em anos chuvosos antes do recente efeito estufa; algumas aves residentes foram caçadas, e os beija-flores das copas talvez estivessem presentes ainda. As espécies de zonas secas, prones à movimentação ou "metapopulacionais", movem os centros de distribuição, camuflando parcialmente a perda de diversidade da mata úmida.

Palavras-chave: aves, conservação, efeito estufa, metapopulações, mata semidecídua, cana-de-açúcar. 


\section{INTRODUCTION}

In central São Paulo State, there was once a belt of semideciduous forest between the evergreen coastal and montane forests, and the interior deciduous woods and savannas (cerrados). Only small patches of the belt persist today, as the region was deforested for agriculture and cities. In 1982, we started studies of birds in a remanescent patch of semideciduous forest, a 230-ha tract (Pagano \& Leitão, 1987; Pagano et al., 1987) on watershed between the basins of the Mogi-Guaçu and Tietê Rivers, at the south edge of the Fazenda São José, border of Rio Claro and Araras townships (22 $21^{\prime} \mathrm{S}$, $47^{\circ} 29^{\prime} \mathrm{W}$ and $675 \mathrm{~m}$ elevation).

Studies of birds of other remnant semideciduous forests include ones done near Campinas and Anhembi (Willis, 1979; Aleixo \& Vielliard, 1995) and northward, near Jardinópolis (Chiarello, 2000). Transitional vegetation approaching the cerrado near São Carlos, just northwest (Marini et al., 1997), has received some study. Two master's theses (Cândido-Júnior, 1991, published 2000; Gondim, 1995) studied edge effects and fruiteating birds in Fazenda São José.

Here we report our bird censuses in and near the Fazenda São José woodlot. Later parts of the study will analyze bird body masses, measurements, molt cycles, ectoparasites, and include detailed observations for understory species captured and banded.

\section{STUDY AREAS AND METHODS}

An originally large forest area was cut into three fragments about 60 years ago (Pagano \& Leitão, 1987). Sugar cane and other agriculture now surround this tract, the southernmost of the fragments. Grassy swales, with ponds in rainy years, cut through the cane fields. A small permanent farm pond near the main road to Araras occupies part of one grassy swale $\left(22^{\circ} 23^{\prime} \mathrm{S}, 47^{\circ} 27^{\prime} \mathrm{W}\right.$, at $650 \mathrm{~m})$. Secondary scrub and woods along the Ribeirão Claro, at $600 \mathrm{~m}$ and $22^{\circ} 20^{\prime} \mathrm{S}, 47^{\circ} 30^{\prime} \mathrm{W}$ on the west edge of the woodlot, partially connect the woodlots. A small side creek, arising in the north edge of the woodlot, provides some wooded swamps on its way west. Just southeast in the forest, an east-west belt of open-understory woods borders another belt of bamboo-crowded understory across the north-south divide of the Mogi-Guaçu and Tietê (border of Rio Claro and Araras townships). Flowering and death of this large bamboo in 1994 left little, except for a small species forming thickets in the central understory. Dense spiny Celtis vines took over in the southern understory, moving in from the wind-damaged south edge of the woodlot. Tall jequitibá (Cariniana estrellensis Kuntz) trees project over the canopy near the divide. Eastward, a cleared swath along an electric power line and a cat-tail marsh cut off two narrow end stretches of the woodlot. The marsh drains southeast toward the Mogi-Guaçu, not southwest to the Tietê as does the Ribeirão Claro.

Several north-south cleared trails, cleared for Pagano's and later studies, cross the woodlot and were used for censuses, as were the road along the west edge near the Ribeirão Claro and one on the south edge along sugar cane fields of Fazenda São João. In the cane field, a dip collected rain water in the strong El Niño years of 1982-83, and we recorded water birds at that time.

We set up trails in a $300 \times 200 \mathrm{~m}$ forest area ( ix 100-m squares) on the divide, by the south edge and sugar cane, in 1983-88; the spiny vines took over later. All banding studies were in the 100-m squares and on a botany trail through the squares. We had hoped to locate bird territories and do behavioral studies, but the wind damage and dense spiny vines complicated our and our students' work. The road along the west edge was closed in 1995; elephant grass grew there, so 13 of 22 later visits were made to the botany trail through the banding area and to the east marsh, rather than to the west road and the botany trail as earlier.

Willis recorded birds at any distance on transect counts, ordinarily from just before dawn to just after midday. He usually walked the west road and back, then the botany trail and back, during about 6 hours from dawn to midday. After the west road was closed, an hour or so at dawn was spent in the east marsh and nearby edges; earlier, these were visited only briefly at dawn and midday for around a half hour. In 1982-1984, when other cross trails were open, he occasionally checked them rather than the main botany trail. Here, as in Willis (1979), we record the number of birds per 100 hours of study, to avoid using space-waste 
decimals. To determine total numbers of birds actually seen, one only need multiply by the number of hours of study divided by 100 .

Banding studies involved 8 mist nets $(12 \mathrm{~m}$ long, of $36 \mathrm{~mm}$ mesh) for about 10 hours daily, along one edge of 100-m squares. We banded on 24 days from 6 Aug. 1983 to 17 June 1984, then 36 days from 12 March 1987 to 16 Aug. 1988. In 3 morning visits in 1985-86 and 12 such visits 1989-2000, 3-5 nets were placed along trails near the road, for ornithology classes. B. S. Ataguile, a student, helped with many captures in 1987-1988; other students also helped. Some 4 hours per visit were of transects along the edge to the east marsh, to the depression in the nearby sugar cane, or on the woodland trails. Half-day visits added 2 hours or so of censuses per visit. Oniki measured the captured birds and checked for ectoparasites, molt, brood patches, and defects.

\section{RESULTS AND DISCUSSION}

\section{Species}

Willis recorded 263 species (Table 1) in the 601.4 hours of censuses (155 visits) 1982-2000.
Xiphorhynchus guttatus, listed by Cândido-Jr. (2000), is not a São Paulo species and may have been a Dendrocolaptes platyrostris. Of these, 67 were birds of surrounding open areas (including distant ponds or marshy zones in the open), 22 of marshes or creeks next to the woods, and 63 edge birds.

Five birds of edges and 5 of the 111 woodland species were vagrants. Subtracting these vagrants and 67 open-area birds, 186 species were birds of the woodlot itself. Of these, 22 were of marsh and water areas, not present in woods studied by Willis (1979). Of the remaining 164 edge and forest species, 12 were not recorded in 1982-86 (152 early species present), 22 not in 1987-92 (144 species present), 32 not 1992-97 (132 species), and 31 not in 1997-2000 (133 species). Four of the species in 1987-92 were not recorded before or since, two in 1992-97, and six in 1997-2000. Some 62 species (considered in a later part of this report) were captured and banded. Canopy species, vagrants, marsh and water birds, and open-area birds were not captured, so that the banding project captured $38 \%$ of the 164 woodlot and border species.

TABLE 1

Birds recorded per 100 hours.

$1=1982-83$ plus 2 visits 1986, $2=1987-92,3=1992-95,4=1997-2000, a=$ banding $1983-86, b=1987-88$, $c=$ end 1988-91, $d=1993-97 . A=$ open areas, $B=$ border, $C=$ creek or marsh, $n=$ migrant from north, $\mathbf{N}=$ winter from north, $\mathbf{S}=$ migrant from south, $\mathrm{S}=$ winter from south, $\mathrm{T}=$ summer breeder, $\mathrm{V}=$ vagrant.

\begin{tabular}{|c|c|c|c|c|c|c|c|c|}
\hline Species & 1 & $\mathbf{a}$ & $\mathbf{b}$ & 2 & c & 3 & d & 4 \\
\hline Crypturellus obsoletus & 22 & 24 & 29 & 27 & 24 & 28 & 50 & 28 \\
\hline Crypturellus parvirostris - A & 7 & 10 & 16 & 20 & 12 & 32 & 20 & 16 \\
\hline Crypturellus tataupa & 9 & 19 & 21 & 25 & 42 & 38 & & 28 \\
\hline Rhynchotus rufescens - A & & & 2 & 1 & 6 & 6 & 10 & 3 \\
\hline Nothura maculosa-A & 4 & 3 & 1 & & & 1 & & 7 \\
\hline Penelope superciliaris & 6 & & & 1 & & 1 & & \\
\hline Dendrocygna viduata - A & 22 & 11 & 8 & & & 1 & & \\
\hline Cairina moschata $-\mathrm{C}$ & 21 & 10 & 4 & 3 & 12 & 7 & 10 & 2 \\
\hline Amazonetta brasiliensis - C & 25 & 16 & 67 & 10 & 6 & 17 & & 20 \\
\hline Nomonyx dominicus - A & 2 & 4 & & & & & & \\
\hline Tringa solitaria - An & 1 & 1 & & & & & & \\
\hline Bartramia longicauda - An & & 10 & & & & & & \\
\hline Gallinago paraguayae - A & 2 & & & & & & & \\
\hline Gallinago undulata - A & & & & & & 8 & 10 & 2 \\
\hline
\end{tabular}


TABLE 1 (Continued)

\begin{tabular}{|c|c|c|c|c|c|c|c|c|}
\hline Species & 1 & $\mathbf{a}$ & $\mathbf{b}$ & 2 & c & 3 & d & 4 \\
\hline Jacana jacana - A & 8 & & 3 & 3 & 6 & 1 & 10 & 3 \\
\hline Vanellus chilensis - A & 19 & 31 & 11 & 16 & 36 & 88 & 300 & 107 \\
\hline Rallus nigricans $-\mathrm{C}$ & 2 & 4 & 6 & 1 & & 3 & & 13 \\
\hline Aramides cajanea - $\mathrm{C}$ & 1 & 1 & 3 & 1 & & 8 & & 8 \\
\hline Aramides saracura $-\mathrm{C}$ & 22 & 3 & & 7 & & 9 & & 13 \\
\hline Porzana albicollis - A & 9 & 9 & 13 & 1 & 12 & 5 & 10 & 7 \\
\hline Laterallus melanophaius - C & 9 & 7 & 6 & 9 & & 5 & 10 & 16 \\
\hline Porphyriops melanops - AS & & 1 & & & & & & \\
\hline Gallinula chloropus - A & 7 & 6 & 4 & 4 & 6 & 21 & 20 & 16 \\
\hline Cariama cristata $-\mathrm{A}$ & 5 & 4 & 5 & 26 & 6 & 29 & 60 & 43 \\
\hline Tachybaptus dominicus - A & 5 & 8 & & & & & & \\
\hline Ardea alba $-\mathrm{A}$ & & & & 3 & & 1 & & 7 \\
\hline Bubulcus ibis - A & & & & 1 & & & & \\
\hline Egretta thula - A & & & & 1 & & & & \\
\hline Butorides striatus $-\mathrm{C}$ & 2 & & 1 & 1 & & 3 & & \\
\hline Syrigma sibilatrix $-\mathrm{A}$ & 3 & 3 & 2 & & 6 & 14 & 50 & 44 \\
\hline Pilherodius pileatus $-\mathrm{C}$ & & & & 1 & & & & \\
\hline Mesembrinibis cayennensis - C & & & & 4 & & 6 & & \\
\hline Coragyps atratus - B & 101 & 109 & 42 & 121 & 72 & 59 & 110 & 104 \\
\hline Cathartes aura & 6 & 1 & & & & & & \\
\hline Elanus leucurus - A & & & 1 & & & & & 2 \\
\hline Leptodon cayanensis & 4 & & & 3 & & 5 & & \\
\hline Accipiter striatus - B & & & & & & & & 1 \\
\hline Buteo albicaudatus - A & & & 2 & & & & & \\
\hline Buteo magnirostris - B & 19 & 9 & 14 & 23 & 12 & 16 & 10 & 35 \\
\hline Buteo brachyurus - B & 3 & & 3 & 3 & & 1 & & \\
\hline Buteogallus meridionalis - A & 1 & 3 & 5 & & 6 & 1 & & 2 \\
\hline Micrastur ruficollis & & 2 & 3 & & & & & \\
\hline Micrastur semitorquatus & & & & & & & & 1 \\
\hline Caracara plancus - B & 25 & 48 & 21 & 20 & 12 & 37 & 20 & 35 \\
\hline Milvago chimachima - A & 8 & & 5 & 4 & 6 & 7 & 10 & 10 \\
\hline Herpetotheres cachinnans - B & 5 & 8 & 5 & 4 & & 7 & & \\
\hline Falco sparverius - A & 1 & 3 & 2 & 3 & 6 & 1 & 10 & 5 \\
\hline Falco femoralis - A & 1 & & 2 & & 6 & 2 & & 3 \\
\hline Otus choliba - B & & 7 & 2 & & & 7 & & 1 \\
\hline Glaucidium brasilianum & 1 & 7 & 15 & 4 & & 7 & & \\
\hline Athene cunicularia - A & 5 & 2 & 5 & & & 8 & & \\
\hline Caprimulgus rufus & & & & & & & & 1 \\
\hline Caprimulgus parvulus - AT & & & & & & 1 & 10 & \\
\hline Nyctidromus albicollis - B & 3 & 5 & 5 & 2 & 30 & 24 & 10 & 13 \\
\hline Hydropsalis torquata - A & & 8 & 16 & 6 & & 47 & 30 & 10 \\
\hline Podager nacunda - A & & & & & & & & 3 \\
\hline Chordeiles minor - AN & & & 4 & & & 3 & & \\
\hline
\end{tabular}

Braz. J. Biol., 62(2): 197-210, 2002 
TABLE 1 (Continued)

\begin{tabular}{|c|c|c|c|c|c|c|c|c|}
\hline Species & 1 & $\mathbf{a}$ & b & 2 & c & 3 & d & 4 \\
\hline Lurocalis semitorquata $-\mathrm{T}$ & & 2 & 2 & & & 9 & & \\
\hline Chaetura meridionalis - AT & 1 & 1 & & & & & & \\
\hline Phaethornis pretrei & 18 & 15 & 22 & 11 & 12 & 13 & 20 & 28 \\
\hline Eupetomena macroura - B & 2 & & 3 & & & 1 & & \\
\hline Florisuga fusca & 10 & 1 & 2 & 3 & & 5 & 10 & 3 \\
\hline Colibri serrirostris - B & 14 & 8 & 16 & 1 & 6 & & & 5 \\
\hline Anthracothorax nigricollis $-\mathrm{T}$ & & & & & & & 10 & \\
\hline Chlorostilbon aureoventris - B & 8 & & 2 & 1 & & 10 & 20 & 5 \\
\hline Thalurania glaucopis & 11 & 7 & 5 & 14 & & 13 & & 3 \\
\hline Amazilia lactea & 34 & 10 & 5 & 7 & & 21 & 30 & 13 \\
\hline Amazilia versicolor & 21 & 8 & 9 & 20 & 6 & 9 & & 3 \\
\hline Aphantochroa cirrochloris & 1 & & & 1 & & & & \\
\hline Leucochloris albicollis - S & & & 1 & & & & & \\
\hline Heliomaster squamosus & 1 & & & 1 & & 2 & & \\
\hline Calliphlox amethystina & 2 & & & & & & & \\
\hline Aratinga leucophthalmus $-\mathrm{S}$ & 15 & & 30 & & 48 & 36 & & 15 \\
\hline Aratinga auricapilla $-\mathrm{V}$ & & & 1 & & & & & \\
\hline Brotogeris chiriri $-\mathrm{V}$ & & & 3 & & & & & \\
\hline Pyrrhura frontalis & 17 & 14 & 30 & & 12 & & & \\
\hline Forpus xanthopterygius - B & 89 & 15 & 58 & 50 & 84 & 38 & 100 & 77 \\
\hline Pionopsitta pileata $-\mathrm{V}$ & & & 1 & & & & & \\
\hline Pionus maximiliani & 109 & 62 & 82 & 50 & 102 & 75 & 80 & 69 \\
\hline Amazona aestiva - V & & & & 1 & & & & \\
\hline Coccyzus melacoryphus - BT & 4 & & 1 & 1 & & & & \\
\hline Coccyzus euleri $-\mathrm{T}$ & 1 & & & & & & & 2 \\
\hline Coccyzus americanus $-\mathrm{N}$ & & & & 1 & & & & \\
\hline Piaya cayana & 65 & 52 & 45 & 50 & 48 & 75 & & 25 \\
\hline Crotophaga ani-B & 64 & 22 & 43 & 20 & 102 & 34 & 60 & 56 \\
\hline Guira guira $-\mathrm{A}$ & 8 & & & 4 & 42 & 8 & 50 & 5 \\
\hline Tapera naevia - B & 18 & 6 & 6 & 11 & 6 & 13 & 20 & 10 \\
\hline Dromococcyx pavoninus $-\mathrm{T}$ & 1 & 2 & 1 & & & 1 & & 3 \\
\hline Columba cayennensis - B & 67 & 16 & 33 & 85 & 96 & 102 & 70 & 69 \\
\hline Columba picazuro - A & 4 & 5 & 5 & 11 & 6 & 19 & & 43 \\
\hline Zenaida auriculata $-\mathrm{A}$ & 134 & 49 & 50 & 27 & 30 & 47 & & 5 \\
\hline Columbina talpacoti - B & 200 & 50 & 36 & 56 & 48 & 47 & 20 & 64 \\
\hline Claravis pretiosa $-\mathrm{T}$ & 13 & & 7 & 7 & & 1 & & \\
\hline Leptotila verreauxi & 159 & 67 & 82 & 174 & 120 & 145 & 100 & 115 \\
\hline Leptotila rufaxilla & & 3 & 1 & 11 & & 5 & & 2 \\
\hline Geotrygon violacea & 2 & & & 1 & & 5 & & 2 \\
\hline Trogon surrucura & 31 & 34 & 33 & 21 & 18 & 23 & & 25 \\
\hline Ceryle torquata $-\mathrm{C}$ & 1 & & & 1 & & & & \\
\hline Chloroceryle amazona $-\mathrm{C}$ & 1 & & & & & 3 & & \\
\hline Chloroceryle americana $-\mathrm{C}$ & 1 & & & 3 & & & & \\
\hline
\end{tabular}


TABLE 1 (Continued)

\begin{tabular}{|c|c|c|c|c|c|c|c|c|}
\hline Species & 1 & $\mathbf{a}$ & $\mathbf{b}$ & 2 & c & 3 & d & 4 \\
\hline Bucco chacuru-A & 1 & & & & & & & \\
\hline Ramphastos toco & & & & & & 3 & & 2 \\
\hline Picumnus albosquamatus & 52 & 36 & 46 & 47 & 36 & 31 & 30 & 30 \\
\hline Melanerpes candidus - B & 5 & 72 & 14 & & 30 & 1 & & 2 \\
\hline Colaptes melanochloros $-\mathrm{B}$ & 16 & 11 & 8 & 16 & & 10 & 10 & 10 \\
\hline Colaptes campestris - A & 19 & 8 & 19 & 27 & 12 & 35 & 30 & 40 \\
\hline Veniliornis passerinus & 33 & 17 & 31 & 24 & & 41 & 30 & 30 \\
\hline Dryicopus lineatus & 22 & 15 & 11 & 26 & 12 & 27 & 10 & 23 \\
\hline Campephilus robustus & 14 & 5 & 9 & 11 & 12 & 8 & & 8 \\
\hline Sittasomus griseicapillus & 35 & 29 & 27 & 24 & 24 & 36 & 70 & 43 \\
\hline Dendrocolaptes platyrostris & 3 & 15 & 4 & 3 & 6 & 8 & & 8 \\
\hline Lepidocolaptes fuscus & 10 & 14 & 14 & & & 1 & & \\
\hline Furnarius rufus - A & & & 7 & 7 & & 15 & 10 & 30 \\
\hline Phacellodomus ferrugineigula - C & 24 & 5 & 2 & 17 & 12 & 16 & 30 & 41 \\
\hline Automolus leucophthalmus & 51 & 45 & 23 & 28 & 18 & 40 & 10 & 15 \\
\hline Lochmias nematura & 1 & 1 & & 1 & & 2 & & 7 \\
\hline Sclerurus scansor & 4 & 1 & 1 & & & 1 & & \\
\hline Xenops rutilans & 4 & 5 & 3 & 3 & & 12 & 20 & 7 \\
\hline Certhiaxis cinnamomea - C & 2 & & 5 & 1 & 6 & 2 & & 3 \\
\hline Certhiaxis vulpina $-\mathrm{C}$ & & & & 3 & & 6 & & \\
\hline Synallaxis spixi-B & 39 & 5 & 3 & 51 & & 56 & 10 & 35 \\
\hline Synallaxis albescens - A & 1 & & 5 & & & & & \\
\hline Synallaxis frontalis - B & 17 & 43 & 44 & 34 & 36 & 40 & 70 & 28 \\
\hline Synallaxis ruficapilla & 116 & 146 & 128 & 118 & 78 & 100 & 110 & 86 \\
\hline Psiloramphus guttatus & 40 & 51 & 34 & 57 & 30 & 36 & 10 & 18 \\
\hline Conopophaga lineata & 103 & 147 & 86 & 161 & 66 & 66 & 100 & 48 \\
\hline Hypoedaleus guttatus - V & & & & 1 & & & 10 & \\
\hline Mackenziaena severa & 83 & 95 & 73 & 67 & 60 & 73 & 80 & 68 \\
\hline Taraba major - B & & 1 & & & & & & 5 \\
\hline Thamnophilus caerulescens & 98 & 80 & 63 & 103 & 54 & 87 & 70 & 63 \\
\hline Thamnophilus ruficapillus - A & & & 1 & & & 2 & & \\
\hline Thamnophilus doliatus - B & 26 & 6 & 12 & 57 & & 46 & 20 & 35 \\
\hline Dysithamnus mentalis & 71 & 105 & 91 & 74 & 24 & 98 & 70 & 92 \\
\hline Herpsilochmus rufimarginatus & 128 & 115 & 87 & 121 & 102 & 125 & 50 & 104 \\
\hline Pyriglena leucoptera & 112 & 210 & 159 & 151 & 132 & 113 & 120 & 112 \\
\hline Drymophila ochropyga & 38 & 66 & 50 & 61 & 72 & 73 & 80 & 72 \\
\hline Drymophila ferruginea & 161 & 135 & 102 & 134 & 144 & 138 & 120 & 91 \\
\hline Phyllomyias fasciatus - BV & 1 & & & & & & & \\
\hline Capsiempis flaveola & 100 & 81 & 82 & 103 & 54 & 131 & 100 & 77 \\
\hline Myiornis auricularis & & 4 & 6 & 4 & 24 & 9 & & 5 \\
\hline Hemitriccus diops & 14 & 32 & 20 & 10 & 18 & 36 & 10 & 30 \\
\hline Hemitriccus nidipendulus - B & 1 & & & & & 1 & & 5 \\
\hline
\end{tabular}


TABLE 1 (Continued)

\begin{tabular}{|c|c|c|c|c|c|c|c|c|}
\hline Species & 1 & a & b & 2 & c & 3 & d & 4 \\
\hline Todirostrum plumbeiceps & 1 & & & 7 & & 3 & & 3 \\
\hline Todirostrum poliocephalum & 37 & 34 & 56 & 53 & 42 & 45 & 50 & 49 \\
\hline Todirostrum cinereum - B & 2 & 8 & 8 & 9 & 12 & 9 & & 21 \\
\hline Serpophaga subcristata - B & 16 & 2 & 2 & 4 & & 7 & & 3 \\
\hline Myiopagis caniceps & 46 & 43 & 42 & 19 & 36 & 25 & & 13 \\
\hline Myiopagis viridicata $-\mathrm{T}$ & 10 & 16 & 20 & 17 & 12 & 17 & 20 & 5 \\
\hline Elaenia chiriquensis - BV & 1 & & & & & & & \\
\hline Elaenia flavogaster - B & 28 & 3 & 9 & 14 & & 21 & 20 & 40 \\
\hline Elaenia spectabilis - CT & 8 & & & 6 & & & & \\
\hline Elaenia obscura - B & 4 & & & 4 & 6 & & & \\
\hline Elaenia mesoleuca $-\mathrm{s}$ & 2 & 1 & & & & 2 & & \\
\hline Elaenia parvirostris $-\mathrm{s}$ & 1 & & & & & & & 3 \\
\hline Phaeomyias murina - BV & 1 & & & & & & & \\
\hline Cnemotriccus bimaculatus - B & 4 & 4 & 3 & & & 17 & 80 & 20 \\
\hline Lathrotriccus euleri & 71 & 70 & 49 & 63 & 36 & 47 & 110 & 30 \\
\hline Myiophobus fasciatus - B & 40 & 23 & 18 & 31 & 30 & 42 & 60 & 36 \\
\hline Platyrhynchus mystaceus & & 1 & 1 & 6 & & 5 & & 5 \\
\hline Tolmomyias sulphurescens & 66 & 54 & 29 & 67 & 24 & 58 & & 48 \\
\hline Contopus cinereus $-\mathrm{s}$ & 11 & 9 & 4 & & & 2 & & 2 \\
\hline Camptostoma obsoletum & 20 & 9 & 11 & 27 & 6 & 32 & 30 & 18 \\
\hline Corythopis delalandi & 1 & & & & & & & \\
\hline Knipolegus cyanirostris - Bs & 2 & 2 & 1 & & & 1 & & 3 \\
\hline Colonia colonus & 60 & 69 & 56 & 26 & 24 & 22 & 40 & 26 \\
\hline Fluvicola leucocephala-C & 13 & 1 & 11 & 6 & 24 & 12 & & 23 \\
\hline Pyrocephalus rubinus - AS & & & & & & & & 3 \\
\hline Xolmis velata $-\mathrm{A}$ & 2 & & & & & 1 & & 3 \\
\hline Xolmis cinerea $-\mathrm{A}$ & & 1 & 6 & & 12 & 2 & 30 & 8 \\
\hline Satrapa icterophrys - Bs & 12 & & & & & 1 & 10 & 8 \\
\hline Gubernetes yetapa - A & 5 & & & & & 3 & 30 & 8 \\
\hline Machetornis rixosus - A & 1 & & & & & 2 & & \\
\hline Tyrannus melancholicus - BT & 26 & 31 & 44 & 50 & 18 & 36 & 60 & 21 \\
\hline Tyrannus savana - As & 1 & & 2 & 7 & & 3 & & 8 \\
\hline Empidonomus varius - BT & 10 & 4 & 10 & 23 & & 22 & 30 & 7 \\
\hline Myiodynastes maculatus - BT & 14 & 28 & 17 & 17 & & 20 & 30 & 13 \\
\hline Pitangus sulphuratus - B & 40 & 17 & 14 & 33 & 6 & 39 & 10 & 48 \\
\hline Myiozetetes similis - $\mathrm{C}$ & 13 & 3 & 11 & 7 & & 18 & 10 & 23 \\
\hline Megarynchus pitangua $-\mathrm{BT}$ & 11 & 10 & 13 & 20 & & 18 & & \\
\hline Myiarchus swainsoni $-\mathrm{T}$ & 13 & 38 & 19 & 9 & & 6 & 30 & 5 \\
\hline Myiarchus ferox-B & 26 & 14 & 17 & 36 & 6 & 30 & 40 & 18 \\
\hline Leptopogon amaurocephalus & 26 & 29 & 15 & 24 & & 21 & 20 & 20 \\
\hline Schiffornis virescens & 7 & 3 & & & & & & \\
\hline Pachyramphus polychopterus $-\mathrm{BT}$ & 14 & & 5 & 24 & & 13 & & 2 \\
\hline
\end{tabular}


TABLE 1 (Continued)

\begin{tabular}{|c|c|c|c|c|c|c|c|c|}
\hline Species & 1 & $\mathbf{a}$ & b & 2 & $\mathbf{c}$ & 3 & d & 4 \\
\hline Pachyramphus viridis - Bs & 2 & & & & & & & \\
\hline Pachyramphus validus - B & 5 & 2 & 3 & 11 & & 12 & 10 & 8 \\
\hline Tityra inquisitor $-\mathrm{s}$ & & & 2 & & & & & \\
\hline Tityra cayana $-\mathrm{T}$ & 1 & 7 & 3 & & & 2 & & 2 \\
\hline Phibalura flavirostris - Bs & 5 & 1 & & & & & & \\
\hline Antilophia galeata $-\mathrm{C}$ & 4 & & & 3 & & & & 2 \\
\hline Chiroxiphia caudata & 81 & 44 & 11 & 54 & 24 & 85 & 40 & 51 \\
\hline Manacus manacus & 8 & 3 & 2 & 7 & 12 & 14 & & 16 \\
\hline Tachycineta leucorrhoa-A & 23 & 92 & 91 & 3 & 66 & 4 & & 26 \\
\hline Progne tapera - AT & 40 & 11 & 14 & 7 & & 19 & 10 & 2 \\
\hline Progne subis - AN & 17 & 4 & 13 & 19 & & & & \\
\hline Progne chalybea-As & 2 & & 2 & & & 1 & & \\
\hline Notiochelidon cyanoleuca - A & 27 & 380 & 194 & 23 & & 119 & 80 & 21 \\
\hline Alopochelidon fucata - As & 2 & 1 & & 2 & & 24 & & 21 \\
\hline Stelgidopteryx ruficollis - B & 48 & 138 & 107 & 14 & 72 & 73 & 90 & 69 \\
\hline Hirundo rustica - AN & 4 & 151 & 53 & & & 1 & & \\
\hline Petrochelidon pyrrhonota - An & & & 30 & 3 & & & & \\
\hline Cyanocorax cristatellus - B & & & & & & 1 & & 3 \\
\hline Troglodytes aedon - B & 16 & 2 & 11 & 3 & 6 & 17 & 10 & 31 \\
\hline Donacobius atricapillus - $\mathrm{C}$ & 11 & 5 & 5 & 7 & 6 & 8 & & 31 \\
\hline Mimus saturninus $-\mathrm{A}$ & 10 & 6 & 13 & 6 & & 12 & 20 & 16 \\
\hline Turdus nigriceps $-\mathrm{s}$ & 1 & 3 & 2 & 3 & & 3 & 10 & \\
\hline Turdus amaurochalinus - B & 35 & 1 & 5 & 20 & 6 & 28 & & 3 \\
\hline Turdus leucomelas - B & 2 & 1 & 11 & 11 & & 28 & & 38 \\
\hline Turdus rufiventris - B & 4 & 3 & 5 & 30 & & 25 & & 12 \\
\hline Turdus albicollis & 10 & 2 & & & & 1 & & \\
\hline Anthus lutescens - A & 7 & 6 & 2 & 3 & 6 & 1 & & \\
\hline Cyclarhis gujanensis & 78 & 58 & 77 & 83 & 54 & 80 & 80 & 51 \\
\hline Vireo olivaceus $-\mathrm{T}$ & 53 & 83 & 42 & 47 & 42 & 40 & 120 & 26 \\
\hline Hylophilus amaurocephalus - B & 14 & & & 1 & & & & 2 \\
\hline Passer domesticus $-\mathrm{A}$ & 3 & & & & & & & \\
\hline Estrilda astrild $-\mathrm{A}$ & 29 & & 13 & & 12 & 14 & & 16 \\
\hline Zonotrichia capensis - B & 123 & 98 & 74 & 60 & 66 & 80 & 180 & 41 \\
\hline Myospiza humeralis - A & 5 & 2 & 9 & & & 8 & 10 & 21 \\
\hline Emberizoides herbicola - A & 2 & 3 & 6 & & 6 & 2 & & 10 \\
\hline Arremon flavirostris & 2 & 4 & & 1 & 6 & & & 10 \\
\hline Arremon semitorquatus & & 1 & & 1 & & & & \\
\hline Donacospiza albifrons - As & 1 & & & & & & & \\
\hline Coryphospingus cucullatus - B & 2 & & 2 & & & & & 2 \\
\hline Haplospiza unicolor $-\mathrm{S}$ & & & & & & 2 & & \\
\hline Tiaris fuliginosa & 10 & 6 & 3 & 6 & & 4 & & 2 \\
\hline Volatinia jacarina $-\mathrm{B}$ & 69 & 12 & 30 & 36 & 24 & 6 & & 74 \\
\hline Sporophila leucoptera-A & & & 2 & & & 1 & & \\
\hline
\end{tabular}


TABLE 1 (Continued)

\begin{tabular}{|c|c|c|c|c|c|c|c|c|}
\hline Species & 1 & $\mathbf{a}$ & b & 2 & c & 3 & d & 4 \\
\hline Sporophila lineola $-\mathrm{BV}$ & 1 & & & & & & & \\
\hline Sporophila caerulescens - B & 110 & 65 & 29 & 46 & & 20 & 20 & 165 \\
\hline Passerina brissoni - BV & & & & 1 & & & & \\
\hline Passerina glaucocaerulea - BS & 1 & 4 & & 1 & 6 & & & \\
\hline Pitylus fuliginosus & 24 & 7 & 3 & 13 & & 2 & 10 & 15 \\
\hline Saltator similis & 34 & 8 & 5 & 21 & & 21 & 10 & 13 \\
\hline Schistochlamys ruficapillus - A & 5 & & & & & & & \\
\hline Hemithraupis ruficapilla & 42 & 57 & 24 & 34 & 6 & 50 & 70 & 33 \\
\hline Nemosia pileata & 12 & 18 & 8 & 9 & & 10 & 20 & \\
\hline Thlypopsis sordida & 58 & 38 & 32 & 43 & 24 & 47 & 20 & 33 \\
\hline Pyrrhocoma ruficeps $-\mathrm{S}$ & 2 & & & & & & & \\
\hline Trichothraupis melanops & 98 & 104 & 67 & 70 & 54 & 77 & 110 & 71 \\
\hline Tachyphonus coronatus & 67 & 31 & 24 & 78 & 36 & 63 & 70 & 46 \\
\hline Habia rubica & 84 & 89 & 52 & 60 & 30 & 89 & & 71 \\
\hline Ramphocelus carbo-B & 44 & 4 & 11 & 58 & & 54 & 60 & 18 \\
\hline Thraupis sayaca-B & 59 & 14 & 28 & 60 & & 48 & 40 & 28 \\
\hline Tangara cayana - B & 42 & 14 & 5 & 48 & & 29 & 20 & 3 \\
\hline Pipraeidea melanonota $-\mathrm{S}$ & 10 & 3 & & & & 2 & & \\
\hline Euphonia chlorotica & 14 & 1 & 9 & 6 & 6 & 3 & & 8 \\
\hline Euphonia violacea $-\mathrm{S}$ & 1 & & 1 & & & 1 & & \\
\hline Euphonia musica $-\mathrm{S}$ & & & & & & & & 2 \\
\hline Dacnis cayana & 13 & & 6 & 6 & 12 & 10 & 20 & 3 \\
\hline Coereba flaveola - B & 53 & 17 & 8 & 19 & & 25 & 60 & 18 \\
\hline Conirostrum speciosum & 53 & 38 & 17 & 37 & & 51 & 50 & 12 \\
\hline Parula pitiayumi & 16 & 5 & 1 & & & & & \\
\hline Geothlypis aequinoctialis - C & 35 & 13 & 17 & 47 & 18 & 64 & 30 & 61 \\
\hline Basileuterus flaveolus & 48 & 56 & 62 & 78 & 96 & 77 & 110 & 66 \\
\hline Basileuterus leucoblepharus & 76 & 8 & & 41 & & 55 & & 36 \\
\hline Basileuterus hypoleucus & 207 & 252 & 148 & 155 & 126 & 150 & 210 & 143 \\
\hline Basileuterus culicivorus $-\mathrm{S}$ & & 3 & & 1 & & 2 & & \\
\hline Pseudoleistes guirahuro - A & 2 & & & 7 & & & & 30 \\
\hline Agelaius cyanopus - C & 4 & & 3 & 3 & 18 & 1 & & 3 \\
\hline Agelaius ruficapillus - A & & & 15 & & & 8 & & 66 \\
\hline Molothrus bonariensis - A & 34 & & & 10 & & 2 & & 2 \\
\hline Cacicus haemorrhous & 8 & & & 9 & & & & \\
\hline Days & 34 & $25^{*}$ & $33^{*}$ & 15 & $5^{*}$ & 20 & $3 *$ & 12 \\
\hline Hours & 166.8 & 100 & 132 & 70.2 & 16.6 & 106 & 10 & 60.7 \\
\hline Night Hours & 0 & + & + & + & + & 8.3 & + & 2.0 \\
\hline
\end{tabular}

* Other days, birds not recorded. Hours of banding not counted. 


\section{Comparison with Campinas woodlot}

In size and forest type, the São José woodlot is rather like the Fazenda Santa Genebra semideciduous woodlot of Campinas, studied in the 1970s by Willis (1979) and in the 1990s by A. Aleixo (Aleixo \& Vielliard, 1995). Considering only the nonvagrant forest and border species, there were 152 species in São José in 1982-86, 144 in 1987-91, 132 in 1992-97, and 133 in 1997-2000. In Santa Genebra, there were 145 such species in the 1970s (147 counting two swifts that fly over) and 117 in the 1990s. Both woodlots lost species gradually.

Other than substitution of Picumnus and Veniliornis for coastal species in Campinas, there were few differences between the woodlots. The kite Ictinia plumbea, antshrike Hypoedaleus, and mixed-flock canopy flycatcher Sirystes sibilator persist in Campinas, while in the 1970's there had been a summering hawk (Harpagus diodon); two owls (Tyto alba, Otus atricapillus); two swifts (Streptoprocne zonaris, Cypseloides fuliginosus); two rare woodcreepers (Dendrocincla turdina, Campylorhamphus falcularius); two flycatchers (open-understory Hemitriccus orbitatus and migrant Elaenia albiceps); and rare, possibly vagrant Dendroica striata (migrant from North America) and Icterus cayanensis (an edge species spreading eastward). In the 1990s, Aleixo added the woodpecker Celeus flavescens plus a possibly vagrant flycatcher (Mionectes rufiventris) and tanager (Tersina viridis), not yet recorded in São José. Campinas gained, much like São José, a few dryforest fruit-eating Aratinga, Amazona, and Ramphastos. Probably present but not seen in the 1970s, Colaptes melanochloros and Turdus rufiventris were also new in Campinas.

By the 1990s, Campinas seemed to have lost Cryturellus obsoletus, Glaucidium, summer Anthracothorax, winter Leucochloris, Coccyzus euleri, Dromococcyx, Sittasomus, Dendrocolaptes, Lepidocolaptes, Automolus, Lochmias, Synallaxis ruficapilla, Mackenziaena, Dysithamnus, Herpsilochmus, Drymophila ferruginea, Capsiempis, Hemitriccus diops, Todirostrum poliocephalum, Myiopagis caniceps, migrant Elaenia mesoleuca, Platyrhynchus, Corythopis, Schiffornis, Hemithraupis, plus winter Euphonia violacea and Basileuterus culicivorus. Several of these were also lost in São José, or no longer reached there in migration.
The Campinas woods lacked several bambooforest upland species throughout, notably Drymophila ochropyga, Psiloramphus, Todirostrum plumbeiceps, and Myiornis. Arremon, Pyriglena, Trogon, and Pyrrhura were also absent, as well as Sclerurus (both the latter disappeared in São José). Several hummingbirds (Aphantochroa, Heliomaster, Calliphlox) although unrecorded in Santa Genebra, were in suburbs there; censusing canopy nectarivores is difficult in Rio Claro and Campinas. Other birds absent in Campinas were Cathartes, Leptodon, Micrastur spp., Herpetotheres (the first two now gone in São José also), and many of the dry-forest species that invaded São José from the northwest (Cyanocorax reached Campinas, as did Ramphastos later).

\section{Lost and new species}

Of 67 open-area species near São José, 22 seemed to disappear in 1997-2000, but open areas were not censused intensively and will very likely still occur with the 45 species recently recorded. Four "lost" species (Thamnophilus ruficapillus, Machetornis, Passer and Schistochlamys) definitely still occur in open areas closer to or in Rio Claro. Cane fields are not suitable for the species of this group, and grassy or bushy swales provide little habitat locally. Progne subis, a northern open-area wintering bird (here present in summer), once roosted in large flocks in city parks in Rio Claro, but has disappeared from the city and region; it was last noted in 1993. A probable southern open-area wintering bird (here present in winter), Donacospiza albifrons, was only noted 8 April 1983 (2 birds together) in marsh scrub near the farm pond, in an El Niño rainy year.

In 1983, a flooded pond inside the cane field attracted several water birds not seen since ( $\mathrm{Ta}$ chybaptus, Nomonyx, northern migrant Tringa, Gallinago paraguayae, southern winter Porphyriops). The first three still occur at times on a large permanent pond in a sizeable eucalyptus grove or "Horto", closer to Rio Claro. The northern migrant Bartramia was spotted in wet cutover cane fields later in 1983, and has not been recorded since. Migrant swallows have been over cane mostly in wet years (Hirundo, Petrochelidon). Dendrocygna was recorded at that time, but later was occasionally seen on the roadside farm pond; hunters probably hunt it there, discouraging recent visits, except at night when we did not visit. During the day, it is 
still common on the Horto pond and a city lake, both protected. Hunters probably rarely check the El Niño pond, hidden in the cane and empty except in wet years.

The four other species that seem to have disappeared recently have always been uncommon in cane areas near Rio Claro, commoner in savannas to the northwest: Buteo albicaudatus (with cane fires), Bucco chacuru, Synallaxis albescens, and Sporophila leucoptera.

Seventeen "new species" of the 67 in open areas probably were not among the 50 species recorded in 1982-86 either because Willis has been checking before dawn mostly in recent years (Rhynchotus, Gallinago undulata, Caprimulgus parvulus, Podager, Chordeiles, Thamnophilus ruficapillus) or because they are rare visitors at the farm pond or just flying over (Ardea spp., Bubulcus, Egretta, Elanus, Buteo albicaudatus, northern migrant Petrochelidon, southern migrant Pyrocephalus, Sporophila leucoptera). The Sporophila, Petrochelidon, Thamnophilus, and Buteo are also unrecorded in recent years (above), as are Bubulcus, Egretta, and Chordeiles.

Two of the 17 new open-area species were perhaps absent 1982-86. Furnarius definitely moved into the farm pond after 1984, perhaps after the muddy roads dried up, allowing it to walk there. Cane areas provide little forage for the species, though houses with lawns are not far south. Agelaius ruficapillus moved to the farm pond marsh, as it is increasing statewide; but cane fields here are rarely suitable for its open-field foraging.

Columba picazuro of dry regions has also invaded São Paulo in recent years (Willis \& Oniki, 1987) and has increased even near Fazenda São José, where it finds food in a few open fields but rarely nests in the few scattered trees, hence not being as common as border nesting $C$. cayennensis. Zenaida of open fields and Columbina of edges seem less usual than during the 1983 El Niño, but both continue common in the city during the last 15 years. As they drink water on open roads in wet years but not in dry, the problem recently may be heat and lack of water at midday in the São José cane fields. Weed seeds may also be less available in dry years. Progne tapera has also become generally less common in recent years and, like other Progne, was perhaps more successful during the wet 1982-84 period; it had roosted in town with P. subis earlier.
Creek and marsh birds were usually present both at the start and end of the studies, though Cairina has become less common in recent dry years. It is still frequent on the protected Horto Pond, as are Butorides and Ceryle (both rare in 1982-84). Aramides saracura appeared on roads more in 1982-84. Elaenia spectabilis seemed to disappear after 1989 and Certhiaxis vulpina after 1995, while Pilherodius was only recorded in 1990 , but censuses were not frequent on the bushy and hard-to-approach creek; these species may have been overlooked as they had never been recorded at the east marsh.

By 1997-2000, some 31 species had disappeared from the woodlot, including 6 border species. Eleven were birds from the south that migrate or winter in the Rio Claro region. Five southern species (Phibalura, Pyrrhocoma, Pipraeidea, Passerina glaucocaerulea, and Basileuterus culicivorus) were registered in the wet El Niño years of 1982-84, with a few winter records later only for Phibalura and glaucocaerulea. Pyrrhocoma, Pipraeidea, and Basileuterus were recorded in the dense understory or canopy of the Rio Claro Horto also mainly during 1982-84. Euphonia violacea was noted infrequently in 1982-92, Leucochloris only in Aug. 1988, Haplospiza only May 1994. They are rare as southern winter visitors in the region. Migrant Pachyramphus viridis, Turdus nigriceps, and Elaenia mesoleuca are also not common locally.

Three summer visitors (Claravis, Coccyzus melacoryphus, Tityra inquisitor) and a northern winter bird (Coccyzus americanus) are also unrecorded recently. Tityra and $C$. americanus are rare in the region, but the other two may have decreased due to recent dry years.

Some other forest and border species that have disappeared since 1982-84 may have been affected by low rainfall in the last 15 years. Arremon semitorquatus of the humid east of the state seems to have been replaced by A. flavirostris of the dry interior. Treetop Parula has dropped in numbers both in São José and in eucalyptuses near Rio Claro, though it seems a dry forest bird and should not have disappeared with droughts. Other treetop birds that join mixed flocks, such as Myiopagis caniceps, Hemitriccus, Conirostrum, and Nemosia also seem less frequent recently. Megarynchus continues in the Horto near Rio Claro, however, and in São José may have little edge habitat except for the hard edges along the cane fields (see below). 
Glaucidium, Pyrrhura, and five other midlevel to understory moist-forest species seem to have disappeared due to loss of the few individuals present earlier: Lepidocolaptes, Sclerurus, Corythopis, Schiffornis, and Turdus albicollis. Cacicus was a river-edge forest species that definitely disappeared from near the east marsh after 1987; it did not survive on Fazenda Barreiro Rico, about 100 km west, after river woods were cut (Willis, 1979; Magalhães, 1999).

Hummingbirds were common mainly in Inga and Tabebuia flowers along the Ribeirão Claro, hence several upper-level species have seldom been recorded of late. Anthracothorax was rare in migratory periods, and Eupetomena and Heliomaster are just rare (more common in the eucalyptus grove off to the south). Study of flower cycles probably would have provided better records. Understory and midlevel Phaethornis, Thalurania, Amazilia lactea, and versicolor were more successfully sampled.

Micrastur ruficollis appeared in 1986-87 and then disappeared. Hunters would not affect these species, although extreme droughts like the La Niña from March to winter of 2000 may have.

Cathartes has disappeared from the Rio Claro region, though still present on rather dry escarpments with better updrafts off to the north (where Sarcorhamphus still occurs). Coragyps declined briefly in 1987, partly due to hunters killing large numbers around a few carcasses. They also may have killed the few Cathartes near Rio Claro. Penelope almost certainly was exterminated by hunters or wandering dogs, first near the roadside banding sites and later in the woods. Various hawks that have disappeared (Leptodon, Buteo brachyurus) may have been shot by occasional hunters, or affected by dry years and low populations. Passerina brissoni is a vagrant in the region due to capture for caging (as Oryzoborus angolensis, Gnorimopsar chopi, Sicalis flaveola), the fate of many macaws and parrots formerly found the Rio Claro region.

Five new forest and border species (Accipiter, Micrastur semitorquatus, Caprimulgus rufus, Ramphastos, Cyanocorax) are still rather rare in the Rio Claro region, and like Taraba major have apparently moved in from dryer and more open regions to the north and west. None have yet been as successful as Columba picazuro, which feeds in open fields. Micrastur do seem to move in and out of local woodlots, as for instance $M$. ruficollis (above). Euphonia musica, a rare winter visitor at forest edges, was recorded only 1997 but was probably present earlier

Some new species may yet disappear and lost species reappear, especially if droughts (possibly linked to greenhouse effects) stop. Many species are able to recolonize (travel-prone species of Willis 1979, metapopulations in more recent works). However, nonmetapopulational forest species like Lepidocolaptes or Schiffornis may be unable to reach the woodlot again. Thus, though border and other species seem to appear and take the place of lost species, the regional diversity will probably decrease with loss of forest species partly concealed by local spread of outside species already present. Entry of new dry-forest species merely moves the centers of distribution of these birds, concealing loss of diversity eastward in moist forest. As houses and intensive agriculture invade the region and water supplies get scarce, even the open-area, border, and water birds will vanish.

\section{Intervening censuses}

In the case of Fazenda São José, we checked birds in intervening years, notably 1987-92 (columns b, 2 and $c$ in Table 1). This allowed one to see if birds absent in the intermediate period had reappeared in the final study periods, rather than being "lost" forever. Eight forest species, seemingly lost in 1987-92, reappeared later: Calliphlox (rare spring), Coccyzus euleri (rare summer), Taraba (invading region), Hemitriccus nidipendulus (rare), Elaenia parvirostris (rare migrant), Satrapa icterophrys (uncommon migrant), Turdus albicollis (rare), and Pipraeidea (rare winter). All but Coccyzus and Turdus are more frequently found at soft or bushy edges, censused less ( 2 of Table 1 ) than earlier. Probably all these species were present in 1987-92, but not recorded; or they were travelprone metapopulations and reinvaded.

This indicates the kind of error one can make in such censuses -8 of 16 species $(50 \%)$ seemingly lost in 1987-92 either moved in again or were actually present throughout. Some 4 species, new in 1987-92 (Micrastur ruficollis, winter Leucochloris, northern winter Coccyzus americanus, summer or migrant Tityra inquisitor), were not sighted later. Some 20 species present both in 198286 and 1987-92 subsequently disappeared. 
In open areas in 1987-92, three uncommon species (Chaetura, Xolmis velata, Gubernetes) of 15 seemingly lost ones (or 20\%) reappeared from 1997 on. Nine rare species previously unrecorded turned up, and four (Bubulcus, Egretta, Buteo albicaudatus, and Petrochelidon) were not seen thereafter, while five (Ardea alba, Chordeiles, Thamnophilus ruficapillus, Agelaius ruficapillus, Sporophila leucoptera) were resighted in later years. Only 2 swallows, present in 1982-86 and 1987-92, vanished later; 12 of 67 open-area species had already been lost soon after the 1982-84 El Niño rains. Open-area species, notably aquatic ones, seemed more directly affected by drought than were forest species.

Of creek and marsh species, only Chloroceryle amazona seemed gone in 1987-92 (reappearing later), while two other species were not reported from 1992 on (Ceryle, Elaenia spectabilis). Pilherodius in 1987-92 was not recorded later, while Mesembrinibis and Certhiaxis vulpina were. As noted above, it was difficult to check these creekside species. Records of these birds seemed mostly unrelated to drought, as creek and marsh water did not dry up as much as did the cane swales or forest.

For the intervening years of 1992-97 (columns 3 and $\mathrm{d}$ in Table 1), 8 forest or border species seemingly lost reappeared later: Colibri, Aphantochroa, Calliphlox, Coccyzus euleri, Taraba, Elaenia spp., Antilophia, Hylophilus, Arremon flavirostris, and Coryphospingus. Thus, 12 of 19 $(63 \%)$ of the birds lost from the preceding period (or earlier) reappeared later. Some 15 other species present up to 1992-97 disappeared later (of these, Turdus albicollis and Pipraeidea were also absent just before). Two species (Anthracothorax and Haplospiza) were only recorded in 1992-97, and Ramphastos and Cyanocorax were new species that also appeared later.

In open areas in 1992-97, only 2 species that had disappeared (Elanus and Pseudoleistes) were noted later, out of 8 species (25\%) seemingly lost after the preceding 5 years. The only new species were Gallinago undulata and Caprimulgus parvulus, noted due to pre-dawn work. Seven species were not seen from 1998 on.

After 1992-97, Butorides and Certhiaxis vulpina seemed to disappear from creeks and marshes. Three species (above) had disappeared by 1992 .

\section{Hard vs. soft edges}

The south forest edge was hard, the forest (though much damaged by wind blowdowns and Celtis vines) abruptly ending at the road and cane field of the neighboring ranch. The west and east edges were either soft or gradual, with scrub and grass, plus additional weeds along the eastern power line swath and cat-tails along the nearby creek. Some species showed a clear difference in censuses because of this, for instance Synallaxis spixi of bushes and tall grass: it was rarely recorded on netting days (abcd in Table 1) along the hard south edge, and regularly on census days (1234 in Table 1) when soft east or west edges were checked. S. frontalis inside shady borders and $S$. ruficapilla in the woods did not show this pattern.

Other birds that showed a preference for soft edges were Aramides saracura and a few other water species or flowering-tree hummingbirds near Ribeirão Claro: Buteo magnirostris; Amazilia; Heliomaster; Tapera; Columbina; Leptotila spp.; Dryocopus; Campephilus; Thamnophilus doliatus; Serpophaga; Elaenia flavogaster and E. obscura; Myiophobus; Camptostoma; Satrapa; Empidonomus; Pitangus; Myiarchus ferox; Pachyramphus (3 spp.); Troglodytes; Turdus amaurochalinus and T. rufiventris; Hylophilus; Volatinia; Sporophila caerulescens; Pitylus (?); Saltator; Thlypopsis; Tachyphonus; Ramphocelus; Thraupis; Tangara; Coereba, and Parula.

Some birds were commoner on regular census days with soft edges, but for other reasons. Several species were found more in swampy uncluttered woods near creeks (not visited on banding days) along the north or west edge: Geotrygon, Lochmias, Sclerurus, 3 manakins, and Basileuterus leucoblepharus. Only the manakins used bushes beyond the forest border. Penelope was absent along the hard cane-field edge due to hunters; it regularly uses similar edges where not hunted.

Birds that seemed somewhat more common (Table 1) along the hard edge were Buteo brachyurus (soars and dives at high edges), Nyctidromus (road by the wood at night), Melanerpes (using roost hole), Synallaxis frontalis (?), Drymophila ochropyga (mainly due to bamboo there), Myiarchus swainsoni (avoids M. ferox?), and Basileuterus flaveolus (avoids B. leucoblepharus?). Birds of 
open areas were often more common there, notably swallows that fly over cane fields.

Some forest birds did enter the cane fields, notably B. flaveolus and $S$. frontalis (see CândidoJr., 2000). Cane cutters reported a banded Pyriglena deep into the cane, near the highway. Willis saw a banded Basileuterus hypoleucus $100 \mathrm{~m}$ out in the cane, near the El Niño pond. Other birds entered the cane fields near the road in the early morning. We recorded such species as Crypturellus parvirostris, Thamnophilus caerulescens, Pyriglena, Conopophaga, Capsiempis, Geothlypis, and Zonotrichia on several occasions. When cane is burned, several hawks, falcons, and caracaras often are present. Bare fields in spring provide a habitat for Vanellus, Syrigma, Xolmis spp., Anthus, and other open-area birds. However, these birds have to leave as the cane grows, and only the Crypturellus regularly move in except for aerial swallows.

\section{Note added in proof}

In 16 more visits $(28 / 7 / 2000$ to $12 / 2 / 2002)$ in and near the São José woodlot, we recorded one or a few Dendrocygna, Bartramia, Bubulcus, Mesembrinibis, Tachybaptus, Cathartes, Leptodon, Buteo brachyurus, Herpetotheres, Glaucidium, Athene, Lurocalis, Caprimulgus parvulus, Chaetura, Eupetomena, Aphantochroa, Calliphlox, Coccyzus melacoryphus, Claravis, Ceryle, Chloroceryle (2 spp.), Thamnophilus ruficapillus, Elaenia obscura and chiriquensis, Megarynchus, Progne chalybea, Turdus nigriceps, Anthus, Passerina glaucocaerulea, Schistochlamys and Sporophila leucoptera and lineola. Thus, instead of 38 species (including 9 border birds) that disappeared from the woodlot in 1997-2000, there were 23 (2) lost in 1997-2002, or 141 species still present. Four new nonforest species (Ardea cocoi flying past, Porphyrula martinica on the cane pond, Myiarchus tyrannulus down Ribeirão Claro, and Sturnella superciliaris near the farm pond) and one hybrid (Sporophila "ardesiaca") were added, for an area total of 266.
Acknowledgments - We thank CEMAVE and IBAMA for bands and permits to work with wild birds. We appreciate equipment from Idea Wild, which made work with data much easier, and field help by students, notably R. Morini and B. S. Ataguile. Publication n. 20 of the Institute for Studies of Nature.

\section{REFERENCES}

ALEIXO, A. \& VIELLIARD, J. M. E., 1995, Composição e dinâmica da avifauna da mata de Santa Genebra, Campinas, São Paulo, Brasil. Rev. Brasil. Zool., 12(3): 493-511.

CÂNDIDO-JÚNIOR, J. F., 1991, Efeito de borda da mata sobre a composição da avifauna em mata residual em Rio Claro, São Paulo. Dissertação de Mestrado, Instituto de Biociências, Unesp, Rio Claro, SP, 110p.

CÂNDIDO-JÚNIOR, J. F., 2000, The edge effect in a forest bird community in Rio Claro, São Paulo State, Brazil. Ararajuba, 8: 9-16.

CHIARELlO, A. G., 2000, Conservation value of a native forest-fragment in a region of extensive agriculture. Rev. Brasil. Biol., 60: 237-247.

GONDIM, M. J. C., 1995, Dispersão de sementes de Trichilia sp. por aves, em uma mata mesófila semidecídua, no município de Rio Claro, SP. Dissertação de Mestrado, Instituto de Biociências, Unesp, Rio Claro, SP, 97p.

MAGALHÃES, J. C. R., 1999, As aves na Fazenda Barreiro Rico. Editôra Plêiade, São Paulo, 215p.

MARINI, M. Â., MOTTA-JUNIOR, J. C., VASCONCELLOS, L. A. S. \& CAVALCANTI, R. B., 1997, Avian body masses from the cerrado region of central Brazil. Ornitol. Neotrop., 8(1): 93-99.

PAGANO, S. N. \& LEITÃO-FILHO, H. F., 1987, Composição florística do estrato arbóreo da mata mesófila semidecídua, no município de Rio Claro (Estado de São Paulo). Rev. Brasil. Bot., 10: 37-47.

PAGANO, S. N., LEITÃO-FILHO, H. F. \& SHEPHERD, G. J., 1987, Estudo fitosociológico em mata mesófila semidecídua no município de Rio Claro (Estado de São Paulo). Rev. Brasil. Bot., 10: 49-61.

WILLIS, E. O., 1979, The composition of avian communities in remanescent woodlots in southern Brazil. Pap. Avulsos Zool., S. Paulo, 33: 1-25.

WILLIS, E. O. \& ONIKI, Y., 1987, Invasion of deforested regions of São Paulo state by the picazuro pigeon, Columba picazuro Temminck, 1813. Ciênc. e Cult., 39(11): 1064-1065. 